\title{
A Delayed Epidemic Model with Pulse Vaccination
}

\author{
Chunjin Wei ${ }^{1,2}$ and Lansun Chen ${ }^{1}$ \\ ${ }^{1}$ Department of Applied Mathematics, Dalian University of Technology, Dalian 116024, China \\ ${ }^{2}$ School of Sciences, Jimei University, Xiamen 361021, China
}

Correspondence should be addressed to Chunjin Wei, chunjinwei92@163.com

Received 14 November 2007; Revised 7 January 2008; Accepted 18 February 2008

Recommended by Leonid Berezansky

A delayed SEIRS epidemic model with pulse vaccination and nonlinear incidence rate is proposed. We analyze the dynamical behaviors of this model and point out that there exists an infectionfree periodic solution which is globally attractive if $R_{1}<1, R_{2}>1$, and the disease is permanent. Our results indicate that a short period of pulse or a large pulse vaccination rate is the sufficient condition for the eradication of the disease. The main feature of this paper is to introduce time delay and impulse into SEIRS model and give pulse vaccination strategies.

Copyright (C) 2008 C. Wei and L. Chen. This is an open access article distributed under the Creative Commons Attribution License, which permits unrestricted use, distribution, and reproduction in any medium, provided the original work is properly cited.

\section{Introduction}

Infectious diseases are usually caused by pathogenic microorganisms, such as bacteria, viruses, parasites, or fungi; the diseases can be spread directly or indirectly. The severe and sudden epidemics of infectious diseases have a great influence on the human life and socioeconomy, which compel scientists to design and implement more effective control and preparedness pro- grams. Pulse vaccination is an effective method to use in attempts to control infectious diseases.

In recent years, epidemic mathematical models of ordinary differential equations have been studied by many authors (e.g., [1-3]). In most of the research literatures, authors always assume that the disease incubation is negligible, therefore, once infected, each susceptible individual becomes infectious instantaneously and later recovers with a temporary acquired immunity. An epidemic model based on these assumptions is customarily called SIR (susceptible, infectious, recovered) model. However, many diseases incubate inside the hosts for a period of time before the hosts become infectious. We assume that a susceptible individual first goes through a latent period after infection before becoming infectious. The resulting model is called SEIRS (susceptible, exposed, infectious, recovered) model. The SEIRS infections disease model is a very important biologic model and has been studied by many authors (e.g., [4-6]). 
Bilinear and standard incidence rates have been frequently used in classical epidemic models [7]. Simple dynamics of these models seem related to such functions. These different incidence rates have been proposed by researchers. Anderson et al. pointed out that standard incidence is more suitable than bilinear incidence [8-10]. Levin et al. have adopted an incidence form like $\beta S^{q} I^{p}$ or $\beta S^{q} I^{p} / N$ which depends on different infective disease and environments [11]. L. S. Chen and J. Chen [12] set forth transmission effect like the saturation effect $\beta S(t) /(1+$ $a S(t))$ as the infection rate. In this paper, we will adopt the infection rate $\beta S(t) /(1+a S(t))$ because it includes the behavioral change and crowding effect of the infective individuals and prevents the unboundedness of the contact rate by choosing suitable parameters.

On the one hand, the newborns of the infectious may already be infected with the disease at birth such as hepatitis and phthisis, and so forth. This is called vertical transmission. On the other hand, some diseases may be spread from one individual to another via horizontal contacting transmission. Some epidemic models with vertical transmission were studied by many authors. However, only a few literatures [13] deal with the analysis of disease with pulse vaccination, vertical and horizontal transmissions.

Most of the research literature on these epidemic models are established by ODE, delayed ODE or impulsive ODE. However, impulsive equations with time delay are not many $[14,15]$. In this paper, we establish a delayed SEIRS epidemic disease model with pulse vaccination and nonlinear incidence rate. We study their dynamic behaviors, establish sufficient condition for disease-eradication, as well as investigate the role of incubation in disease transmission. The main feature of this paper is to introduce time delay and pulse vaccination into epidemic model and obtain some important qualitative properties with valid pulse vaccination strategy.

The organization of this paper is as follows. In the next section, we introduce the delayed SEIRS model with pulse vaccination. To prove our main results, we also give several definitions, notations, and lemmas. In Section 3, we investigate the dynamic behavior of the model with nonlinear incidence and the sufficient condition is obtained for the global attractivity of infection-free periodic solution and the permanence of the model. In the final section, we try to interpret our mathematical results in terms of their ecological implication and also point out some future research directions.

\section{Model formulation and preliminary}

In the following model, we study a population that is partitioned into four classes, the susceptible, exposed, infectious, and recovered, with sizes denoted by S, E, I, and R, respectively, and we consider pulse vaccination strategy in the delayed SEIRS epidemic model with nonlinear incidence rate $\beta(S /(1+a S)) I$, the following mathematical model is formulated:

$$
\begin{aligned}
& S^{\prime}(t)=A-\beta \frac{S(t) I(t)}{1+a S(t)}-\mu S(t)-(1-p) \mu I(t)+\alpha R(t), \quad t \neq n T, \\
& E^{\prime}(t)=\beta \frac{S(t) I(t)}{1+a S(t)}-\beta e^{-\mu \tau} \frac{S(t-\tau) I(t-\tau)}{1+a S(t-\tau)}-\mu E(t)+(1-p) \mu I(t), \quad t \neq n T, \\
& I^{\prime}(t)=\beta e^{-\mu \tau} \frac{S(t-\tau) I(t-\tau)}{1+a S(t-\tau)}-(r+d+\mu) I(t), \quad t \neq n T, \\
& R^{\prime}(t)=r I(t)-\mu R(t)-\alpha R(t), \quad t \neq n T,
\end{aligned}
$$




$$
\begin{aligned}
S\left(t^{+}\right) & =(1-\theta) S(t), \quad t=n T, n=1,2, \ldots \\
E\left(t^{+}\right) & =E(t), \quad t=n T, n=1,2, \ldots \\
I\left(t^{+}\right) & =I(t), \quad t=n T, n=1,2, \ldots \\
R\left(t^{+}\right) & =R(t)+\theta S(t), \quad t=n T, n=1,2, \ldots
\end{aligned}
$$

Here, all coefficients are positive constants, $A$ denotes the influx or recruitment of the susceptible and the exposed. The death rate for disease and physical disease rate are $d$ and $\mu$, respectively. $r$ is the recovery rate of infectious individual. $\theta(0<\theta<1)$ is the proportion of those vaccinated successfully, which is called impulsive vaccination rate. $\tau$ is the latent period of the disease. Consider the death of exposed individuals during latent period of disease, that is, $\beta e^{-\mu \tau}(S(t-\tau) I(t-\tau) /(1+a S(t-\tau)))$ term. The disease is propagated both vertically and horizontally, $p \mu I(0<p<1)$ is the number of newborns of infectious who transfer to the susceptible class, and $(1-p) \mu I$ is the number of newborns of the infectious who are infected vertically.

The total population size $N(t)=S(t)+E(t)+I(t)+R(t)$ can be determined by the differential equation

$$
N^{\prime}(t)=A-\mu N(t)-d I(t)
$$

which is derived by adding all equations in system (2.1). So we have $A-(\mu+d) N(t) \leq N^{\prime}(t) \leq$ $A-\mu N(t)$. It follows that

$$
\frac{A}{\mu+d} \leq \lim _{t \rightarrow \infty} \inf N(t) \leq \lim _{t \rightarrow \infty} \sup N(t) \leq \frac{A}{\mu}
$$
model:

Before going to any detail, we simplify model (2.1) and mainly discuss the following

$$
\begin{aligned}
S^{\prime}(t) & =A-\beta \frac{S(t) I(t)}{1+a S(t)}-\mu S(t)-(1-p) \mu I(t)+\alpha R(t), \quad t \neq n T, \\
I^{\prime}(t) & =\beta e^{-\mu \tau} \frac{S(t-\tau) I(t-\tau)}{1+a S(t-\tau)}-(r+d+\mu) I(t), \quad t \neq n T, \\
R^{\prime}(t) & =r I(t)-\mu R(t)-\alpha R(t), \quad t \neq n T, \\
N^{\prime}(t) & =A-\mu N(t)-d I(t), \quad t \neq n T, \\
S\left(t^{+}\right) & =(1-\theta) S(t), \quad t=n T, n=1,2, \ldots \\
I\left(t^{+}\right) & =I(t), \quad t=n T, n=1,2, \ldots \\
R\left(t^{+}\right) & =R(t)+\theta S(t), \quad t=n T, n=1,2, \ldots \\
N\left(t^{+}\right) & =N(t), \quad t=n T, n=1,2, \ldots
\end{aligned}
$$

The initial condition of (2.4) is given as

$$
\phi(\xi)=\left(\phi_{1}(\xi), \phi_{2}(\xi), \phi_{3}(\xi), \phi_{4}(\xi)\right) \in C_{+}, \quad \phi_{i}(0)>0, i=1,2,3,4,
$$


where $C_{+}=C\left([-\tau, 0], R_{+}^{4}\right)$. From biological considerations, we discuss system (2.4) in the closed set

$$
\Omega=\left\{(S, I, R, N) \in R_{+}^{4} \mid 0 \leq S+I+R \leq \frac{A}{\mu}, N \leq \frac{A}{\mu}\right\},
$$

where $R_{+}^{4}$ denotes the nonnegative cone of $R^{4}$ including its lower dimensional faces. It is easy to show that $\Omega$ is positively invariant with respect to (2.4).

Before starting our main results, we give the following lemmas.

Lemma 2.1 (see [16]). Consider the following delay differential equation:

$$
x^{\prime}(t)=a x(t-\tau)-b x(t),
$$

where $a, b, \tau>0$ and $x(t)>0$ for $t \in[-\tau, 0]$. The following hold:

(i) if $a<b$, then $\lim _{t \rightarrow \infty} x(t)=0$,

(ii) if $a>b$, then $\lim _{t \rightarrow \infty} x(t)=+\infty$.

Lemma 2.2. Consider the following impulsive differential equations:

$$
\begin{aligned}
u^{\prime}(t) & =a-b u(t), \quad t \neq n T, \\
u\left(t^{+}\right) & =(1-\theta) u(t), \quad t=n T, n \in N,
\end{aligned}
$$

where $a>0, b>0,0<\theta<1$. Then there exists a unique positive periodic solution of (2.8):

$$
\tilde{u}_{e}(t)=\frac{a}{b}+\left(u^{*}-\frac{a}{b}\right) e^{-b(t-k T)}, \quad k T<t<(k+1) T,
$$

which is globally asymptotically stable, where $u^{*}=a(1-\theta)\left(1-e^{-b T}\right) / b\left(1-(1-\theta) e^{-b T}\right)$.

\section{Global attractivity of infection-free periodic solution}

In this section, we study the existence of the infection-free periodic solution of system (2.4), in which infectious individuals are entirely absent from the population permanently, that is, $I(t)=0$ for all $t \geq 0$. Under this condition, system (2.4) becomes the following impulsive system without delay:

$$
\begin{aligned}
S^{\prime}(t) & =A-\mu S(t)+\alpha R(t), \quad t \neq n T, \\
R^{\prime}(t) & =\mu R(t)-\alpha R(t), \quad t \neq n T, \\
N^{\prime}(t) & =A-\mu N(t), \quad t \neq n T, \\
S\left(t^{+}\right) & =(1-\theta) S(t), \quad t=n T, n=1,2, \ldots \\
R\left(t^{+}\right) & =R(t)+\theta S(t), \quad t=n T, n=1,2, \ldots \\
N\left(t^{+}\right) & =N(t), \quad t=n T, n=1,2, \ldots
\end{aligned}
$$


From the third and sixth equations of system (3.1), we have $\lim _{t \rightarrow \infty} N(t)=A / \mu$. Further, if $I(t)=0$, it follows that $\lim _{t \rightarrow \infty} E(t)=0$ from the second and sixth equations of system (2.1). In the following, we show that the susceptible population $S$ and recovered population $R$ oscillate with period $T$, in synchronization with the periodic impulsive vaccination. Consider the following limit system of system (3.1):

$$
\begin{aligned}
R(t) & =\frac{A}{\mu}-S(t), \\
S^{\prime}(t) & =(\alpha+\mu)\left(\frac{A}{\mu}-S(t)\right), \quad t \neq n T, \\
S\left(t^{+}\right) & =(1-\theta) S(t), \quad t=n T, n \in N .
\end{aligned}
$$

By Lemma 2.2, we know that the periodic solution of system (3.2),

$$
\tilde{S}_{e}(t)=\frac{A}{\mu}+\left(S^{*}-\frac{A}{\mu}\right) e^{-(\alpha+\mu)(t-n T)}, \quad n T<t \leq(n+1) T,
$$

is globally asymptotically stable, where $S^{*}=A(1-\theta)\left(1-e^{-(\alpha+\mu) T}\right) / \mu\left(1-(1-\theta) e^{-(\alpha+\mu) T}\right)$.

Denote $R_{1}=\beta e^{-\mu \tau} \delta /(1+a \delta)(r+d+\mu)$, where $\delta=A\left(1-e^{-(\alpha+\mu) T}\right) / \mu\left(1-(1-\theta) e^{-(\alpha+\mu) T}\right)$.

Theorem 3.1. If $R_{1}<1$, then the infection-free periodic solution $\left(\widetilde{S}_{e}(t), 0, A / \mu-\widetilde{S}_{e}(t), A / \mu\right)$ of system (2.4) is globally attractive.

Proof. Since $R_{1}<1$, we can choose $\varepsilon>0$ small enough such that

$$
\frac{\beta e^{-\mu \tau}(\delta+\varepsilon)}{1+a(\delta+\varepsilon)}<r+d+\mu
$$

From the first equation of system (2.4), it follows that $S^{\prime}(t) \leq(\alpha+\mu)((A / \mu)-S(t))$. Thus consider the following comparison impulsive differential system:

$$
\begin{aligned}
& z^{\prime}(t)=(\alpha+\mu)\left(\frac{A}{\mu}-z(t)\right), \quad t \neq n T, \\
& z\left(t^{+}\right)=(1-\theta) z(t), \quad t=n T, n \in N .
\end{aligned}
$$

By (3.2), we know that the periodic solution of system (3.5),

$$
\tilde{z}_{e}(t)=\widetilde{S}_{e}(t)=\frac{A}{\mu}+\left(S^{*}-\frac{A}{\mu}\right) e^{-(\alpha+\mu)(t-n T)}, \quad n T<t \leq(n+1) T,
$$

is globally asymptotically stable, where $S^{*}=A(1-\theta)\left(1-e^{-(\alpha+\mu) T}\right) / \mu\left(1-(1-\theta) e^{-(\alpha+\mu) T}\right)$. Let $(S(t), I(t), R(t), N(t))$ be the solution of system (2.4) with initial condition (2.5) and $S\left(0^{+}\right)=$ $S_{0}>0, z(t)$ be the solution of system (3.5) with initial value $z\left(0^{+}\right)=S_{0}$. By the comparison theorem for impulsive differential equations [17], there exists an integer $n_{1}>0$ such that

$$
S(t)<z(t)<\tilde{z}_{e}(t)+\varepsilon, \quad n T<t \leq(n+1) T, n>n_{1},
$$


that is,

$$
S(t)<\tilde{z}_{e}(t)+\varepsilon \leq \frac{A\left(1-e^{-(\alpha+\mu) T}\right)}{\mu\left(1-(1-\theta) e^{-(\alpha+\mu) T}\right)}+\varepsilon \dot{\doteq} \eta .
$$

Further, from the second equation of system (2.4), we have that, for all $t>n T+\tau, n>n_{1}$,

$$
I^{\prime}(t) \leq \frac{\beta \eta e^{-\mu \tau}}{1+a \eta} I(t-\tau)-(r+d+\mu) I(t)
$$

Consider the following comparison equation:

$$
y^{\prime}(t)=\frac{\beta \eta e^{-\mu \tau}}{1+a \eta} y(t-\tau)-(r+d+\mu) y(t)
$$

From (3.4), we have that

$$
\frac{\beta \eta e^{-\mu \tau}}{1+a \eta}<(r+d+\mu)
$$

According to Lemma 2.1, we obtain that $\lim _{t \rightarrow \infty} y(t)=0$.

Set $(S(t), I(t), R(t), N(t))$ be the solution of system (2.4) with initial condition (2.5) and $I(\xi)=\phi(\xi)>0(\xi \in[-\tau, 0]), y(t)$ be the solution of $(3.10)$ with initial condition $y(\xi)=\phi(\xi)>$ $0(\xi \in[-\tau, 0])$. By the comparison theorem in differential equation and the positivity of solution (with $I(t) \geq 0$ ), we have that

$$
\lim _{t \rightarrow \infty} I(t)=0 .
$$

Therefore, for any $\varepsilon_{1}>0$ (sufficiently small), there exists an $n_{2}\left(n_{2} T>n_{1} T+\tau\right)$ such that $0<I(t)<\varepsilon_{1}$ for all $t>n_{2} T$. By the fourth equation of system (2.4), we have

$$
N^{\prime}(t)>A-\mu N(t)-d \varepsilon_{1} \quad \text { for } t>n_{2} T .
$$

Consider the following comparison equation: $z_{1}^{\prime}(t)=\left(A-d \varepsilon_{1}\right)-\mu z_{1}(t)$.

It is clear that $\lim _{t \rightarrow \infty} z_{1}(t)=\left(A-d \varepsilon_{1}\right) / \mu$; by the comparison theorem, we have that there exists an integer $n_{3}>n_{2}$ such that for all $t>n_{3} T, N(t) \geq\left(A-d \varepsilon_{1}\right) / \mu-\varepsilon_{1}$. Since $\varepsilon_{1}$ is arbitrarily small, we have

$$
\lim _{t \rightarrow \infty} N(t)=\frac{A}{\mu} .
$$

It follows from (3.12) and (3.14) that, there exists $n_{4}>n_{3}$ such that

$$
I(t)<\varepsilon_{1}, \quad N(t)>\frac{A}{\mu}-\varepsilon_{1} \quad \text { for } t>n_{4} T .
$$

From the second equation of system (2.1), we have

$$
E^{\prime}(t) \leq\left(\frac{A \beta \varepsilon_{1}}{\mu+a A}+(1-p) \mu \varepsilon_{1}\right)-\mu E(t) \quad \text { for } t>n_{4} T .
$$

It is easy to obtain that there exists an $n_{5}>n_{4}$ such that

$$
E(t)<\delta_{1}+\varepsilon_{1} \text { for } t>n_{5} T,
$$


where $\delta_{1}=\left(A \beta \varepsilon_{1}+(\mu+a A)(1-p) \mu \varepsilon_{1}\right) / \mu(\mu+A a)$. So from the first equation of system (2.4), we have

$$
S^{\prime}(t) \geq\left(A+\frac{\alpha A}{\mu}-(1-p) \mu \varepsilon_{1}-\alpha \delta_{1}-3 \alpha \varepsilon_{1}\right)-\left(\beta \varepsilon_{1}+\mu+\alpha\right) S(t) .
$$

Consider the following comparison impulsive differential equations for $t>n_{5} T$ and $n>n_{5}$ :

$$
\begin{aligned}
& u^{\prime}(t)=\left(A+\frac{\alpha A}{\mu}-(1-p) \mu \varepsilon_{1}-\alpha \delta_{1}-3 \alpha \varepsilon_{1}\right)-\left(\beta \varepsilon_{1}+\mu+\alpha\right) u(t), \quad t \neq n T, \\
& u\left(t^{+}\right)=(1-\theta) u(t), \quad t=n T, n \in N .
\end{aligned}
$$

By Lemma 2.2, we know that the periodic solution of system (3.19) is

$$
\tilde{u}_{e}(t)=\Theta+\left(u^{*}-\Theta\right) e^{-\left(\alpha+\mu+\beta \varepsilon_{1}\right)(t-n T)}, \quad n T<t \leq(n+1) T,
$$

which is globally asymptotically stable, where

$$
\begin{aligned}
\Theta & =\frac{A+A \alpha / \mu-\alpha \delta_{1}-3 \alpha \varepsilon_{1}-(1-p) \mu \varepsilon_{1}}{\alpha+\mu+\beta \varepsilon_{1}}, \\
u^{*} & =\Theta \frac{(1-\theta)\left(1-e^{-\left(\alpha+\mu+\beta \varepsilon_{1}\right) T}\right)}{1-(1-\theta) e^{-\left(\alpha+\mu+\beta \varepsilon_{1}\right) T}} .
\end{aligned}
$$

By using the comparison theorem of impulsive differential equation [17], there exists an $n_{6}>n_{5}$ such that

$$
S(t)>\tilde{u}_{e}(t)-\varepsilon_{1}, \quad n T<t \leq(n+1) T, n>n_{6} .
$$

Let $\varepsilon_{1} \rightarrow 0$, then it follows from (3.8) and (3.22) that

$$
\tilde{S}_{e}(t)=\frac{A}{\mu}\left(1-\frac{\theta e^{-(\alpha+\mu)(t-n T)}}{1-(1-\theta) e^{-(\alpha+\mu) T}}\right), \quad n T<t \leq(n+1) T,
$$

is globally attractive, that is,

$$
\lim _{t \rightarrow \infty} S(t)=\widetilde{S}_{e}(t)
$$

By the positivity of $E(t)$ and sufficiently small $\varepsilon_{1}$, it follows from (3.17) that

$$
\lim _{t \rightarrow \infty} E(t)=0 \text {. }
$$

From the restriction $N(t)=S(t)+E(t)+I(t)+R(t)$, we have $\lim _{t \rightarrow \infty} R(t)=A / \mu-\widetilde{S}_{e}(t)$. Therefore, the infection-free periodic solution $\left(\widetilde{S}_{e}(t), 0, A / \mu-\widetilde{S}_{e}(t), A / \mu\right)$ is globally attractive. This completes the proof.

Corollary 3.2. In system (2.4), the following states are true.

(i) If $A \beta e^{-\mu \tau}<(r+d+\mu)(\mu+A a)$, then infection-free periodic solution $\left(\widetilde{S}_{e}(t), 0, A / \mu-\right.$ $\left.\widetilde{S}_{e}(t), A / \mu\right)$ is globally attractive. 
(ii) If $A \beta e^{-\mu \tau}>(r+d+\mu)(\mu+A a)$ and $T<T^{*}$, then infection-free periodic solution $\left(\widetilde{S}_{e}(t), 0, A / \mu-\widetilde{S}_{e}(t), A / \mu\right)$ is globally attractive, where $T^{*}=1 /(\alpha+\mu) \ln (1+(\theta(r+d+$ $\mu) \mu) /\left(A \beta e^{-\mu \tau}-a A(r+d+\mu)-(r+d+\mu) \mu\right)$.

(iii) If $\theta>\theta^{*}$, then infection-free periodic solution $\left(\widetilde{S}_{e}(t), 0, A / \mu-\widetilde{S}_{e}(t), A / \mu\right)$ is globally attractive, where $\theta^{*}=(r+d \mu) e^{-(\alpha+\mu) T}-(r+d+\mu) \mu+\left(A \beta e^{-\mu \tau}-a(r+d+\mu) A\right)\left(1-e^{-(\alpha+\mu) T}\right) / \mu(r+$ $d+\mu) e^{-(\alpha+\mu) T}$.

Theorem 3.1 determines the global attractivity of system (2.4) in $\Omega$ for the case $R_{1}<1$. From Corollary 3.2, we can see that a short pulse periodic (with $T$ ) or a large pulse vaccination rate (with $\theta$ ) is the sufficient condition for the global attractivity of infection-free periodic solution $\left(\widetilde{S}_{e}(t), 0, A / \mu-\widetilde{S}_{e}(t), A / \mu\right)$.

\section{Permanence}

In this section, it is noted that the disease is endemic if the infectious population persists above a certain threshold for sufficiently large time. The endemicity of the disease can be well captured and studied through the notion of uniform persistence and permanence.

Definition 4.1. System (2.4) is said to be uniformly persistent if there exists an $m>0$ (independent of the initial data) such that every solution $(S(t), I(t), R(t), N(t))$ with initial conditions (2.5) of system (2.4) satisfies

$$
\lim _{t \rightarrow \infty} \inf S(t) \geq m, \quad \lim _{t \rightarrow \infty} \inf I(t) \geq m, \quad \lim _{t \rightarrow \infty} \inf R(t) \geq m, \quad \lim _{t \rightarrow \infty} \inf N(t) \geq m .
$$

Definition 4.2. System (2.4) is said to be permanent if there exists a compact region $\Omega_{0} \in$ int $\Omega$ such that every solution of system (2.4) with initial data (2.5) will eventually enter and remain in region $\Omega_{0}$.

Denote

$$
\begin{aligned}
R_{2} & =\frac{\left(\beta e^{-\mu \tau} /(r+d+\mu)-a\right) A(1-\theta)\left(1-e^{-\mu T}\right)}{\mu\left(1-(1-\theta) e^{-\mu T}\right)}, \\
I^{*} & =\frac{A \mu\left(R_{2}-1\right)}{A \beta+(1-p) \mu^{2} R_{2}} .
\end{aligned}
$$

Theorem 4.3. If $R_{2}>1$, then there exists a positive constant $m$ such that each positive solution $(S(t), I(t), R(t), N(t))$ of system (2.4) satisfies $I(t) \geq m$ for $t$ large enough.

Proof. Note that the second equation of system (2.4) can be rewritten as follows:

$$
I^{\prime}(t)=I(t)\left(\beta e^{-\mu \tau} \frac{S(t)}{1+a S(t)}-(r+d+\mu)\right)-\beta e^{-\mu \tau} \frac{d}{d t} \int_{t-\tau}^{t} \frac{S(u) I(u)}{1+a S(u)} d u .
$$

Define

$$
V(t)=I(t)+\beta e^{-\mu \tau} \int_{t-\tau}^{t} \frac{S(u)}{1+a S(u)} I(u) d u
$$


According to (4.3), we calculate the derivative of $V$ along the solution of (2.4):

$$
\begin{aligned}
V^{\prime}(t) & =I(t)\left(\beta e^{-\mu \tau} \frac{S(t)}{1+a S(t)}-(r+d+\mu)\right) \\
& =(r+d+\mu) I(t)\left(\frac{\beta e^{-\mu \tau} S(t)}{(r+d+\mu)(1+a S(t))}-1\right) .
\end{aligned}
$$

Since $R_{2}>1$, then $I^{*}>0$ and there exists sufficiently small $\varepsilon>0$ such that

$$
\frac{\beta e^{-\mu \tau} \sigma}{(r+d+\mu)(1+a \sigma)}>1
$$

where

$$
\sigma=\frac{\left(A-(1-p) \mu I^{*}\right)(1-\theta)\left(1-e^{-\left(\beta I^{*}+\mu\right) T}\right)}{\left(\beta I^{*}+\mu\right)\left(1-(1-\theta) e^{-\left(\beta I^{*}+\mu\right) T}\right)}-\varepsilon>0 .
$$

We claim that for any $t_{0}>0$, it is impossible that $I(t)<I^{*}$ for all $t \geq t_{0}$. Otherwise, there is a $t_{0}>0$ such that $I(t)<I^{*}$ for all $t \geq t_{0}$. It follows from the first equation of (2.4) that we have

$$
S^{\prime}(t)>\left(A-(1-p) \mu I^{*}\right)-\left(\beta I^{*}+\mu\right) S(t)
$$

Consider the following comparison impulsive system for $t \geq t_{0}$ :

$$
\begin{aligned}
v^{\prime}(t) & =\left(A-(1-p) \mu I^{*}\right)-\left(\beta I^{*}+\mu\right) v(t), \quad t \neq n T, \\
v\left(t^{+}\right) & =(1-\theta) v(t), \quad t=n T, n \in N .
\end{aligned}
$$

According to Lemma 2.2, we obtain that

$$
\tilde{v}_{e}(t)=\frac{A-(1-p) \mu I^{*}}{\beta I^{*}+\mu}+\left(v^{*}-\frac{A-(1-p) \mu I^{*}}{\beta I^{*}+\mu}\right) e^{-\left(\beta I^{*}+\mu\right)(t-n T)}, \quad n T<t \leq(n+1) T,
$$

is the unique globally asymptotically stable positive periodic solution, where $v^{*}=(A-(1-$ p) $\left.\mu I^{*}\right)(1-\theta)\left(1-e^{-\left(\beta I^{*}+\mu\right) T}\right) /\left(\beta I^{*}+\mu\right)\left(1-(1-\theta) e^{-\left(\beta I^{*}+\mu\right) T}\right)$.

By the comparison theorem for impulsive differential equation [17], we know that there exists $t_{1}>t_{0}+\tau$ such that the following inequality holds for $t>t_{1}$ :

$$
S(t)>\widetilde{v}_{e}(t)-\varepsilon
$$

Thus

$$
S(t)>v^{*}-\varepsilon \doteq \sigma>0 \quad \text { for } t \geq t_{1} .
$$

From (4.6), we have $\beta e^{-\mu \tau} \sigma /(r+d+\mu)(1+a \sigma)>1$. By (4.5) and (4.12), we have

$$
V^{\prime}(t)>(r+d+\mu) I(t)\left(\frac{\beta e^{-\mu \tau} \sigma}{(r+d+\mu)(1+a \sigma)}-1\right) \text { for } t \geq t_{1}
$$


Let

$$
I_{l}=\min _{t \in\left[t_{1}, t_{1}+\tau\right]} I(t) .
$$

We will show that $I(t) \geq I_{l}$ for all $t \geq t_{1}$. Otherwise, there is a $T_{0}>0$ such that $I(t) \geq I_{l}$ for $t \in\left[t_{1}, t_{1}+\tau+T_{0}\right], I\left(t_{1}+\tau+T_{0}\right)=I_{l}$, and $I^{\prime}\left(t_{1}+\tau+T_{0}\right) \leq 0$. However, the second equation of systems (2.4) and (4.12) imply that

$$
I^{\prime}\left(t_{1}+\tau+T_{0}\right) \geq(r+d+\mu) I_{l}\left(\frac{\beta e^{-\mu \tau} \sigma}{(r+d+\mu)(1+a \sigma)}-1\right)>0 .
$$

This is a contradiction, thus, $I(t) \geq I_{l}$ for all $t \geq t_{1}$. As a consequence, (4.13) leads to

$$
V^{\prime}(t)>(r+d+\mu) I_{l}\left(\frac{\beta e^{-\mu \tau} \sigma}{(r+d+\mu)(1+a \sigma)}-1\right)>0 \quad \text { for } t \geq t_{1}
$$

which implies that $V(t) \rightarrow \infty$ as $t \rightarrow \infty$. This contradicts with $V(t) \leq(A / \mu)\left(1+A \tau \beta e^{-\mu \tau} / \mu\right)$. Hence, for any $t_{0}>0$, the inequality $I(t)<I^{*}$ cannot hold for all $t \geq t_{0}$. Next, we are left to consider two cases:

(1) $I(t) \geq I^{*}$ for $t$ large enough;

(2) $I(t)$ oscillates about $I^{*}$ for $t$ large enough.

It is clear that if $I(t) \geq I^{*}$ for $t$ large enough, then our aim is obtained. So we only need consider the case (2).

Let

$$
m=\min \left\{\frac{I^{*}}{2}, I^{*} e^{-(r+d+\mu) \tau}\right\} .
$$

In the following, we will show that $I(t) \geq m$ for $t$ large enough, let $t^{*}>0$ and $\iota>0$ satisfy $I\left(t^{*}\right)=I\left(\iota+t^{*}\right)=I^{*}$, and $I(t)<I^{*}$ for $t^{*}<t<t^{*}+\iota$, where $t^{*}$ is sufficiently large such that $S(t)>\sigma$ for $t^{*}<t<t^{*}+t$, we can conclude that $I(t)$ is uniformly continuous since the positive solution of (2.4) is ultimately bounded and $I(t)$ is not affected by impulsive effects. Hence there exists a constant $T_{1}\left(0<T_{1}<\tau\right.$, and $T_{1}$ is independent of the choice if $\left.t^{*}\right)$ such that $I(t)>I^{*} / 2$ for $t^{*} \leq t \leq t^{*}+T_{1}$. If $\iota \leq T_{1}$, our aim is obtained. If $T_{1}<\iota \leq \tau$, since $I^{\prime}(t)>-(r+d+\mu) I(t)$, and $I\left(t^{*}\right)=I^{*}$, it is obvious that $I(t) \geq I^{*} e^{-(r+d+\mu) \tau}$ for $t^{*}<t<t^{*}+\iota$. If $\iota>\tau$; by the second equation of (2.4), we obtain $I(t) \geq I^{*} e^{-(r+d+\mu) \tau}$ for $t^{*}<t<t^{*}+\tau$. The same arguments can be continued, we can obtain $I(t) \geq I^{*} e^{-(r+d+\mu) \tau}$ for $t^{*}+\tau<t<t^{*}+\iota$. Since the interval $\left[t^{*}, t^{*}+\iota\right]$ is arbitrarily chosen, we can conclude that $I(t) \geq m$ for $t$ large enough. Based on the above discussions, the choice of $m$ is independent of the positive solution of (2.4), and we have proved that any positive solution of (2.4) satisfies $I(t) \geq m$ for all sufficiently large $t$. This completes the proof.

Theorem 4.4. If $R_{2}>1$, then the system (2.4) is permanent.

Proof. Suppose that $(S(t), I(t), R(t), N(t))$ be any solution of (2.4). From the first equation of (2.4), we have

$$
S^{\prime}(t) \geq p A-\left(\frac{\beta A}{\mu}+\mu\right) S(t)
$$


Similarly, we have

$$
\lim _{t \rightarrow \infty} S(t) \geq q
$$

where

$$
q=\frac{p A \mu}{\beta A+\mu^{2}} \frac{(1-\theta)\left(1-e^{-(\beta(A / \mu)+\mu) T}\right)}{1-(1-\theta) e^{-(\beta(A / \mu)+\mu) T}}-\varepsilon .
$$

By Theorem 4.3, the third equation of (2.4) becomes

$$
R^{\prime}(t) \geq r m-(\mu+\alpha) R(t) .
$$

It is easy to obtain that

$$
R(t) \geq \frac{r m}{\mu+\alpha}-\varepsilon \doteq \omega
$$

Set

$$
\Omega_{0}=\left\{(S, I, R, N) \mid q \leq S, m \leq I, \omega \leq R, S+I+R \leq \frac{A}{\mu}, \frac{A}{\mu+d}-\varepsilon \leq N \leq \frac{A}{\mu}\right\} .
$$

By Theorem 3.1 and above discussions, we know that the set $\Omega_{0}$ is a global attractor in $\Omega$ and, of course, every solution of system (2.4) with initial condition (2.5) will eventually enter and remain in region $\Omega_{0}$. Hence system (2.4) is permanent. This completes the proof.

Denote

$$
\begin{aligned}
& T_{*}=-\frac{1}{\mu} \ln \frac{1}{1-\theta}\left(1-\frac{\theta\left(\beta e^{-\mu \tau} /(r+d+\mu)-a\right) A}{\left(\beta e^{-\mu \tau} /(r+d+\mu)-a\right) A-\mu}\right), \\
& \theta_{*}=1-\frac{\mu e^{\mu T}}{A\left(\beta e^{-\mu \tau} /(r+d+\mu)-a\right)\left(e^{\mu T}-1\right)+\mu} .
\end{aligned}
$$

Corollary 4.5. The following results are true.

(1) If $T>T_{*}$, then system (2.4) is permanent.

(2) If $\theta<\theta_{*}$, then system (2.4) is permanent.

\section{Discussion}

In this paper, we introduce the delayed SEIRS epidemic model with pulse vaccination and nonlinear incidence rate of the form $\beta(S(t) I(t) /(1+a S(t)))$. As a result, it is observed that nonlinear incidence, the latent period of disease, pulse vaccination rate, and pulse vaccination period bring effects on the dynamics of our model. Theorems 3.1 and 4.4 show that $R_{1}\langle 1, \theta\rangle$ $\theta^{*}$, or $T<T^{*}$ implies that the disease will be eradicated, whereas $R_{2}>1, \theta<\theta_{*}$, or $T>T_{*}$ implies that the disease will be epidemic. Our results indicate that a short pulse time or a large pulse vaccinate rate will lead to eradication of the disease. In this paper, we only discuss $R_{1}<1$ and $R_{2}>1$, but for closed interval $\left[R_{1}, R_{2}\right]$, the dynamical behaviors of system (2.4) have not been studied, that is, the threshold parameter for the reproducing number between the eradication and the permanence of the disease has not been studied, which will be left in the future research. 


\section{Acknowledgment}

This work was supported by National Natural Science Foundation of China (10771179).

\section{References}

[1] G. Pang and L. Chen, "A delayed SIRS epidemic model with pulse vaccination," Chaos, Solitons $\mathcal{E}$ Fractals, vol. 34, no. 5, pp. 1629-1635, 2007.

[2] B. Shulgin, L. Stone, and Z. Agur, "Pulse vaccination strategy in the SIR epidemic model," Bulletin of Mathematical Biology, vol. 60, pp. 1123-1148, 1998.

[3] G. Li and Z. Jin, "Global stability of a SEIR epidemic model with infectious force in latent, infected and immune period," Chaos, Solitons and Fractals, vol. 25, no. 5, pp. 1177-1184, 2005.

[4] K. L. Cooke and P. van den Driessche, "Analysis of an SEIRS epidemic model with two delays," Journal of Mathematical Biology, vol. 35, no. 2, pp. 240-260, 1996.

[5] W. Wang, "Global behavior of an SEIRS epidemic model with time delays," Applied Mathematics Letters, vol. 15, no. 4, pp. 423-428, 2002.

[6] X. Meng, L. Chen, and H. Cheng, "Two profitless delays for the SEIRS epidemic disease model with nonlinear incidence and pulse vaccination," Applied Mathematics and Computation, vol. 186, no. 1, pp. 516-529, 2007.

[7] H. W. Hethcote, “The mathematics of infectious diseases," SIAM Review, vol. 42, no. 4, pp. 599-653, 2000.

[8] R. Anderson and R. May, Population Biological of Infectious Disease, Springer, Heidelberg, Germany, 1982.

[9] R. Anderson and R. May, Infectious Disease of Human: Dynamics and Control, Oxford University Press, Oxford, UK, 1991.

[10] M. D. Jong, O. Diekmann, and J. Heesterbeek, "How dose transmisson depend on population size," in Human Infectious Disease. Epidemic Models, D. Mollison, Ed., pp. 84-94, Cambridge University Press, Cambridge, UK, 1995.

[11] W.-M. Liu, S. A. Levin, and Y. Iwasa, "Influence of nonlinear incidence rates upon the behavior of SIRS epidemiological models," Journal of Mathematical Biology, vol. 23, no. 2, pp. 187-204, 1986.

[12] L. S. Chen and J. Chen, Nonlinear Biological Dynamics System, Scientific Press, China, 1993.

[13] S. Tang and L. Chen, "The effect of seasonal harvesting on stage-structured population models," Journal of Mathematical Biology, vol. 48, no. 4, pp. 357-374, 2004.

[14] J. Yan, A. Zhao, and J. J. Nieto, "Existence and global attractivity of positive periodic solution of periodic single-species impulsive Lotka-Volterra systems," Mathematical and Computer Modelling, vol. 40, no. 5-6, pp. 509-518, 2004.

[15] B. Zhang and Y. Liu, "Global attractivity for certain impulsive delay differential equations," Nonlinear Analysis. Theory, Methods \& Applications, vol. 52, no. 3, pp. 725-736, 2003.

[16] Y. Kuang, Delay Differential Equations with Applications in Population Dynamics, vol. 191 of Mathematics in Science and Engineering, Academic Press, Boston, Mass, USA, 1993.

[17] V. Lakshmikantham, D. D. Bainov, and P. S. Simeonov, Theory of Impulsive Differential Equations, vol. 6 of Series in Modern Applied Mathematics, World Scientific, Singapore, 1989. 


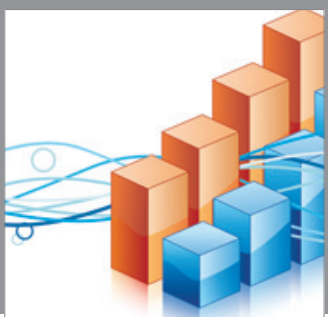

Advances in

Operations Research

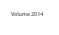

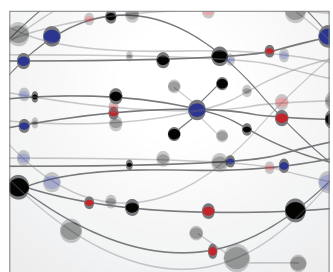

\section{The Scientific} World Journal
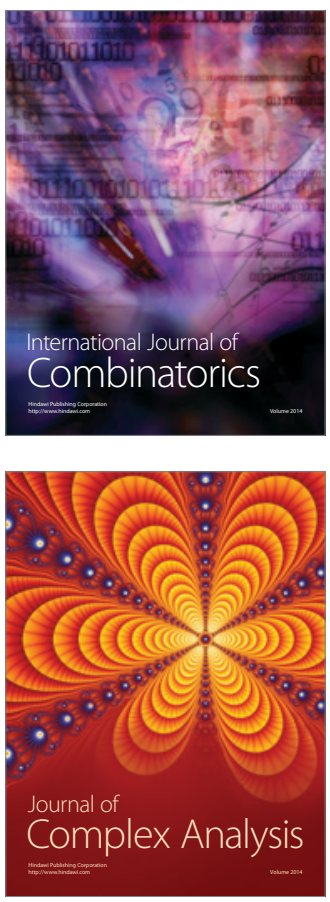

International Journal of

Mathematics and

Mathematical

Sciences
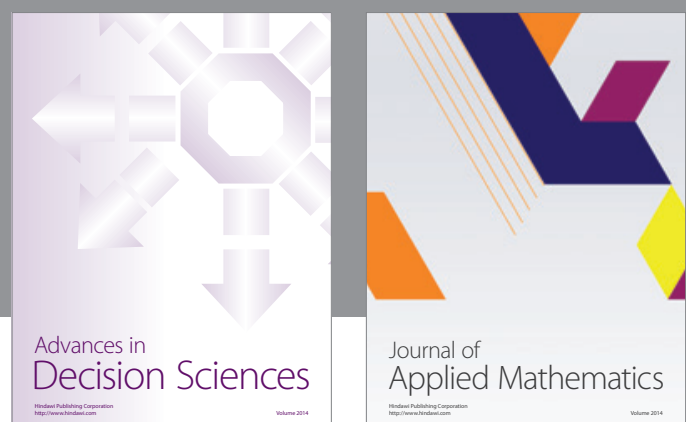

Journal of

Applied Mathematics
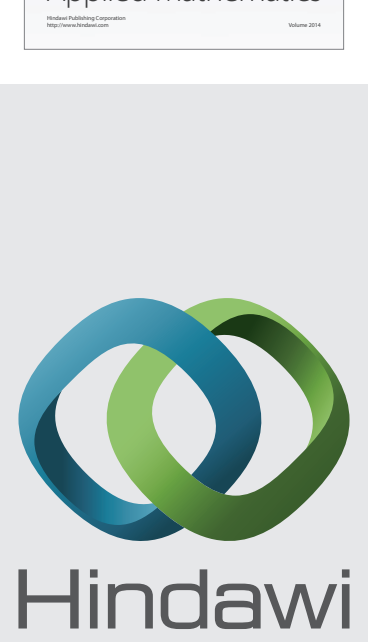

Submit your manuscripts at http://www.hindawi.com
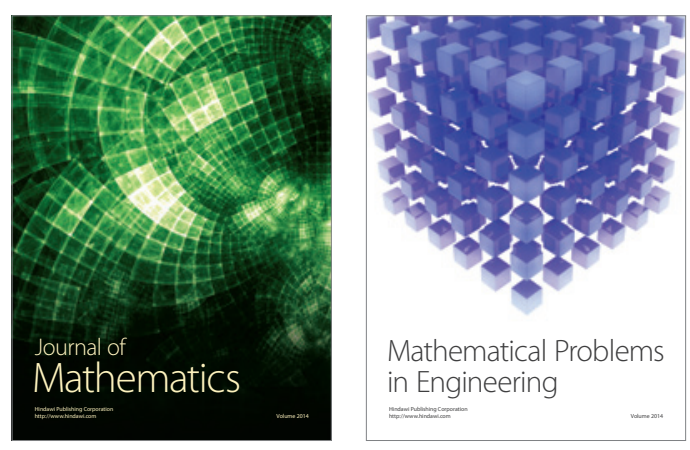

Mathematical Problems in Engineering
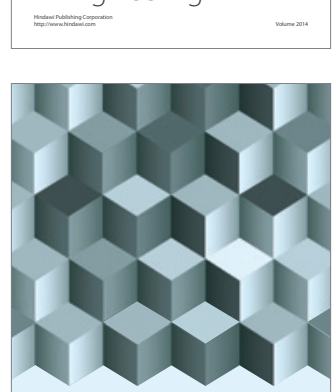

Journal of

Function Spaces
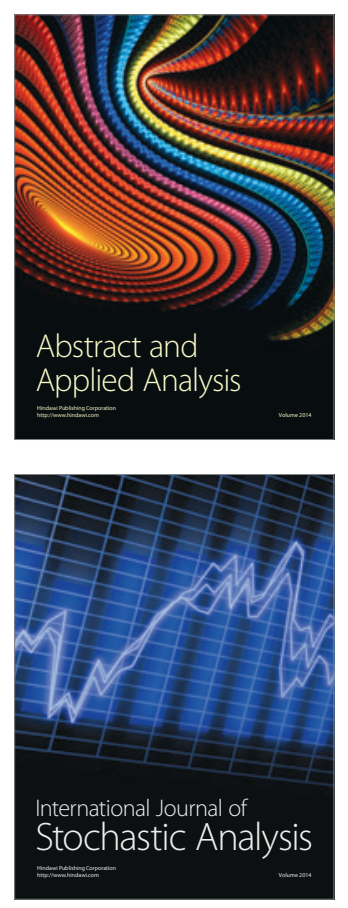

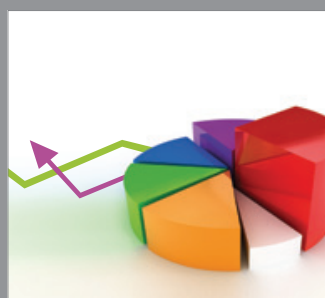

ournal of

Probability and Statistics

Promensencen
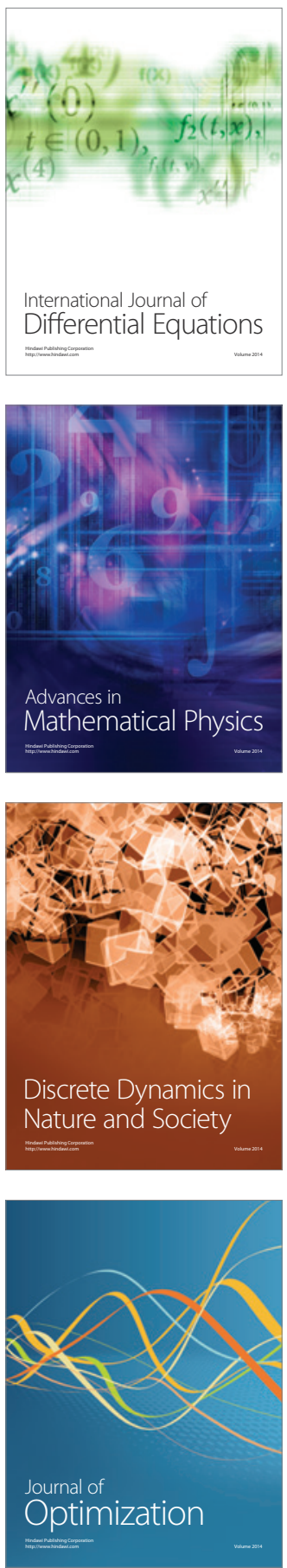